\title{
Os determinantes da eficiência na Atenção Primária à Saúde dos municípios paulistas: um modelo georreferenciado
}

\section{I ${ }^{1}$ Tiago Silveira Gontijo, ${ }^{2}$ I|ka Afonso Reis |}

Resumo:Esta pesquisa teve por objetivo mensurar a eficiência dos gastos em Atenção Primária à Saúde (APS) dos municípios paulistas e avaliar sua correlação com a densidade demográfica e o tamanho populacional dos municípios, assim como com sua localização geográfica e seu PIB per capita. Realizou-se uma avaliação ex post, quantitativa e descritiva acerca da eficiência do gasto em APS para uma amostra dos municípios paulistas ( $\mathrm{n}=509)$, considerando os dados do ano de 2015. A presente pesquisa fez uso de um modelo de Data Envelopment Analysis com Supereficiência para detectar e remover outliers. Por fim, verificou-se a magnitude da relação existente entre os escores de eficiência calculados e as variáveis não discricionárias. Verificou-se que a média dos escores de eficiência após a exclusão dos municípios supereficientes (outliers) tornou-se maior (IC99\%: 0.127; 0.213). Em média, os municípios paulistas são moderadamente ineficientes no tocante aos gastos com APS $(\theta=0.488)$. Destaca-se que os municípios situados na faixa Leste do Estado de SP tendem a ter maiores níveis de eficiência e que a eficiência é inversamente proporcional ao tamanho populacional. O SUS possui notado subfinanciamento, mas há espaço para melhorias na gestáo dos gastos e aumento da eficiência no serviço prestado.

> Palavras-chave: Atenção Primária à Saúde; eficiência; escores robustos; determinantes.

\footnotetext{
1 Universidade Federal de Minas Gerais. Belo Horizonte-MG, Brasil (tsgontijo@hotmail.com)

ORCID: 0000-0003-2636-899X

${ }^{2}$ Universidade Federal de Minas Gerais. Belo Horizonte-MG, Brasil (ilka@est.ufmg.br).

ORCID: 0000-0001-7199-8590
}

Recebido em: 28/05/2019 Aprovado em: 22/11/2019 Revisado em: 29/03/2021 


\section{Introdução}

Inspirado pelo National Health Service (NHS) do Reino Unido, o Sistema Único de Saúde (SUS) está em vias de completar trinta anos (COSTA; BAHIA; SCHEFFER, 2013). Se, por um lado, o SUS representa um importante avanço constitucional, que prevê que "a saúde é um direito de todos e dever do Estado" (BRASIL, 1988, Art. 196), por outro, ele possui importantes desafios, a saber: o congelamento dos gastos governamentais pelos próximos vinte anos (MÔNICA et al., 2017); o impacto da alternância de poder nas políticas para a saúde (GOMES, 2014); a complexidade em se levar a saúde para 5.570 municípios brasileiros com características culturais e sociais díspares (SANTOA; CAMPOS, 2015), além de uma série de determinantes econômicos, representados pelo subfinanciamento do sistema (PAIM, 2013).

Apesar dos desafios apresentados, a Constituição Federal determina, através da Lei Complementar no 141/2012, que a União investirá em saúde o valor aplicado no ano anterior acrescido da variação nominal do produto interno bruto (PIB) dos dois anos anteriores. Já os estados terão de aplicar 12\% de suas receitas, e os municípios, 15\% (BRASIL, 2012). Diante do exposto, a notada escassez de recursos, principalmente no âmbito municipal, bem como a crescente demanda por serviços hospitalares no Brasil têm despertado o desenvolvimento de pesquisas que buscam mensurar a eficiência dos gastos públicos em saúde (VIEIRA, 2008).

É importante destacar que o sistema de saúde adotado no Brasil é hierarquizado através de serviços de saúde organizados por faixas de complexidade, em uma organização piramidal (Paim, 2008). Ainda, segundo a Portaria no 4.279/2010, os serviços de saúde estruturam-se por meio de arranjos produtivos conformados segundo as densidades tecnológicas singulares, variando do nível de menor densidade - Atenção Primária à Saúde (APS), ao de densidade tecnológica intermediária - Atenção Secundária à Saúde, até o de maior densidade tecnológica - Atenção Terciária à Saúde (BRASIL, 2010).

A APS é a principal porta de entrada aos serviços de saúde e funciona como um filtro capaz de organizar o fluxo dos serviços na rede do SUS, dos mais simples aos mais complexos (BRASIL, 2011), contempla açôes que abrangem a promoção e a proteção da saúde, a prevenção de agravos, o diagnóstico, o tratamento, a reabilitação, a redução de danos e a manutenção da saúde e precisa, portanto, funcionar com 
eficiência. No entanto, a APS é caracterizada pelo excesso de demanda, ausência de profissionais (MITRE; ANDRADE; COTTA, 2012).

O cálculo da eficiência é um tema amplamente discutido na literatura (LIU et al., 2013; EMOUZNEJAD; YANG, 2017). Quando aplicado à gestão de serviços de saúde, permite determinar a performance dos municípios (ZHU, 2014). Segundo Jamas e Pollitt (2000), os principais métodos de estimação da eficiência empregados são a Data Envelopment Analysis (DEA) e a Stochastic Frontier Analysis (SFA). Destaca-se ainda que os modelos da classe DEA têm sido os mais utilizados pela literatura científica para tal fim (GUERRINI; ROMANO; CAMPEDELLI, 2013; WORTHINGTON, 2014).

Diante do exposto, formula-se a seguinte questão: qual é o impacto da (in) eficiência dos gastos em atenção primária à saúde dos municípios paulistas? Para responder a esta pergunta, o presente artigo tem por objetivo propor um modelo para avaliar a eficiência dos serviços municipais de saúde. Especificamente, pretende-se: (i) Determinar, através do modelo DEA-Supereficiência, quais são os municípios classificados como outliers; (ii) Calcular os escores de eficiência para o ano de 2015 e verificar o impacto da exclusão dos outliers na eficiência global; (iii) Examinar a distribuição espacial dos escores de eficiência relativa à APS; e (iv) Verificar a influência da densidade demográfica, latitude, longitude, PIB per capita e tamanho populacional na eficiência dos municípios paulistas.

\section{Métodos}

Eficiência é um conceito relativo que compara o que uma firma (Decision Making Unit - DMU) produziu (outputs), dados os recursos disponíveis (inputs), com o que poderia ser produzido com os mesmos recursos (Zhu, 2014). A DEA é uma técnica não paramétrica de mensuração da eficiência, e que utiliza uma fronteira determinística de produçáo para definir a maior quantidade possível de outputs produzidos por um dado vetor de inputs. A difusão da DEA se deu, sobretudo, a partir dos trabalhos seminais de Charnes, Cooper e Rhodes (1978) e Banker, Charnes e Cooper (1984). A presente pesquisa utilizou os municípios do estado de São Paulo como DMU. A próxima etapa na utilização da DEA é a escolha dos inputs e outputs do modelo. Esta é uma etapa fundamental, pois os escores de eficiência são diretamente influenciados por essas variáveis (COOK; TONE; ZHU, 2014). Porém, 
há que se frisar que não há consenso na literatura sobre quais inputs e outputs devam ser empregados na avaliação da eficiência em Atenção Básica em Saúde (CABRAL et al., 2016; BRAGA; FERREIRA; BRAGA, 2015; OZCAN, 2014).

Com o objetivo de exemplificar a variabilidade de modelos utilizados pela literatura, destaca-se por sua vez, que Cabral et al. (2016) utilizaram os inputs: recurso ABS; número de agentes comunitários de saúde, número de unidades básicas de saúde instaladas e número de equipes relativas à Estratégia Saúde da Família implantadas (per capita). Os outputs adotados foram: percentual de gestantes maiores de 20 anos, nascidos vivos cujas mães realizaram sete ou mais consultas pré-natais, média de cobertura vacinal, proporção de internaçóes hospitalares por condições não sensíveis à atenção ambulatorial da população de 1-5 anos e proporção de internações hospitalares por condições não sensíveis à atenção ambulatorial da população de 60 anos ou mais.

Duarte et al. (2016), por sua vez, adotaram como inputs o total da despesa com a função Saúde e escolheram, múltiplos outputs, a saber: quantidade aprovada de produção ambulatorial realizada pelo SUS, de doses aplicadas em açóes de vacinação por município considerando população-alvo, de visitas das equipes de Saúde da Família (ESF) e agentes comunitários de saúde às famílias e de famílias que cadastradas nas equipes de Saúde da Família. Por fim, mas não menos importante, destaca-se que Braga, Ferreira e Braga (2015) utilizaram o número de equipes de saúde e gastos municipais com atenção básica como inputs e o número de famílias acompanhadas pelo Programa de Saúde da Família e número de visitas domiciliares como outputs.

Neste artigo, considerou-se um input similar ao utilizado nos trabalhos supracitados: o gasto per capita em Atenção Primária em Saúde (em reais), coletado junto à Secretaria do Tesouro Nacional (STN). Quanto aos outputs, foram considerados os indicadores que monitoram a ampliação do acesso à Atenção Básica de Saúde (expressos em escala numérica que varia de 0 a 100), os quais constam no rol de diretrizes, objetivos, metas e indicadores 2013-2015, pactuado pelo Conselho Nacional de Saúde (BRASIL, 2014). Desta forma, os seis outputs adotados foram: (i) Cobertura populacional estimada pelas equipes de atenção básica; (ii) Proporção de internações por condiçôes sensíveis à atenção básica; (iii) Cobertura de acompanhamento das condicionalidades de saúde do Programa Bolsa Família (PSF); (iv) Cobertura populacional estimada pelas equipes básicas de Saúde Bucal; 
(v) Proporçâo da ação coletiva de escovaçâo dental supervisionada; e (vi) Proporção média de exodontia em relação aos procedimentos.

Os dados foram coletados no sítio do Ministério da Saúde (BRASIL, 2014). Destaca-se que, para manter a coerência com as metas estipuladas, no cálculo dos escores de eficiência foram considerados como outputs o inverso da proporção de internações por condições sensíveis à atenção básica e o inverso da proporção de exodontia em relação aos procedimentos, pois se espera que, quanto mais eficiente for a gestão, menores serão esses indicadores, ou seja, maiores serão os inversos deles. De posse destas variáveis, realizou-se uma avaliação ex post, quantitativa e descritiva, considerando os dados do ano de 2015, o mais recente com informaçóes disponíveis.

Basicamente, o método DEA consiste em um problema de programação linear, na qual se admite que $k$ DMU utilizem uma tecnologia comum para transformar um vetor $m$-dimensional de inputs $x^{k}=\left(x_{1}^{k}, \ldots, x_{m}^{k}\right) \in \Re_{+}^{m}$ em um vetor de n-dimensional outputs $y^{k}=\left(y_{1}^{k}, \ldots, y_{n}^{k}\right) \in \mathfrak{R}_{+}^{n}$.. Assim, define-se o conjunto de possibilidades de produção $(T)$, formado pelos planos viáveis $(x, y) T=$, de forma que: $T=\left\{(x, y) \in \mathfrak{R}_{+}^{m} x \mathfrak{R}_{+}^{n} \mid x\right.$ pode produzir $\left.y\right\}$. T é, portanto, o conjunto de valores $(x, y)$ para os quais existe um $k$ tal que $(x, y)=\left(x^{k}, y^{k}\right)$, ou $\quad$ seja: $\quad T=\left\{(x, y) \in \Re_{+}^{m} x \Re_{+}^{n} \mid \exists k \in\{1, \ldots, K\}:(x, y)=\left(x^{k}, y^{k}\right)\right\}$ (BOGETOFT; OTTO, 2010).

A orientação de modelos DEA pode ser a outputs ou a inputs e depende da possibilidade de controle das variáveis produzidas e dos objetivos dos gestores das DMU (COOK; TONE; ZHU, 2014). No caso em questão, considerou-se a orientação a inputs, uma vez que ela é a mais utilizada pela literatura internacional. A abordagem orientada para inputs pressupôe basicamente que os municípios classificados como eficientes são aqueles, que, mantendo o nível de resultados constantes, conseguiram minimizar o uso dos recursos. Uma vantagem da orientação a inputs reside no fato de que os outputs são variáveis exógenas e que os governos locais têm um controle significativo sobre os insumos.

Além disso, admitiu-se o pressuposto de Variable returns to scale (VRS), pois, segundo Ferreira e Gomes (2012, p. 194), a hipótese de retornos constantes de escala “[...] somente é apropriada quando todas as DMU estão operando em escala ótima. Competição imperfeita, restriçooes financeiras, dentre outras causas, podem levar uma DMU a não operar em escala de produção ótima”. 
A presente pesquisa fez uso do modelo clássico DEA-VRS (BANKER; CHARNES; COOPER, 1984) para o cálculo do escore de eficiência $(\Theta)$ da i-ésima $\operatorname{DMU}\left(\theta_{\mathrm{i}}\right)$, conforme pode ser visualizado em (1).

$$
\begin{gathered}
\theta_{\mathrm{i}}=\operatorname{Max} \sum_{\mathrm{i}=1}^{\mathrm{m}} \mathrm{u}_{\mathrm{i}} \mathrm{y}_{\mathrm{ij}}+\mathrm{u}_{0} \\
\sum_{\mathrm{i}=1}^{\mathrm{m}} \mathrm{u}_{\mathrm{i}} \mathrm{y}_{\mathrm{ij}}-\sum_{\mathrm{i}=1}^{\mathrm{s}} \mathrm{v}_{\mathrm{i}} \mathrm{x}_{\mathrm{ij}}+\mathrm{u}_{0} \leq 0 ; \forall_{\mathrm{j}}=1, \ldots, \mathrm{N} ; \sum_{\mathrm{i}=1}^{\mathrm{s}} \mathrm{v}_{\mathrm{i}} \mathrm{x}_{\mathrm{ij}}=1 ; \mathrm{u}_{\mathrm{i}} \geq 0 \forall_{\mathrm{i}}=1, \mathrm{~m} ; \mathrm{v}_{\mathrm{i}} \geq 0 \forall_{\mathrm{i}}=1, \mathrm{~m}
\end{gathered}
$$

onde $u_{i}$ e $v_{i}$ são, respectivamente, os pesos referentes aos outputs e inputs de cada DMU. A variável $\mathrm{u}_{0}$ é irrestrita em sinal e denota a classificação dos rendimentos de escala: se o rendimento é decrescente, $\mathrm{u}_{0}<0$; se o rendimento for constante, então $\mathrm{u}_{0}=0$. Por fim, para rendimentos crescentes, tem-se que $\mathrm{u}_{0}>0$. No caso de $\theta_{\mathrm{i}}=1$, maior valor possível, o município é considerado como totalmente eficiente, ao passo que, se $\theta_{\mathrm{i}}=0$, o município é classificado como totalmente ineficiente. Logo, $\left(1-\theta_{\mathrm{i}}\right)$ denota o grau de ineficiência do i-ésimo município analisado.

Embora a técnica DEA-VRS seja flexível quanto à adequação aos dados, pois se admitem inputs e outputs com diferentes unidades de medida, Banker et al. (2004) recomendam que o número de DMU seja igual a, no mínimo, três vezes a soma do número de inputs e outputs, pois a pequena quantidade de DMU em comparação com o número de inputs e outputs diminui o poder de discriminação da DEA (COOK; TONE; ZHU, 2014).

Destaca-se que os escores de eficiência gerados pela DEA-VRS são sensíveis à presença de outliers. Isso porque a ocorrência de inputs ínfimos ou outputs muito acima do esperado para alguma DMU acarretaria níveis mais baixos de eficiência para as DMU restantes, o que pode levar a uma subestimativa substancial dos escores gerais de eficiência. Mais precisamente, a DEA-VRS é baseada no (extremo) conceito de fronteira, composto por um número relativamente pequeno de observaçôes, onde uma única DMU distante da média pode empurrar a fronteira e, portanto, deslocar artificialmente os requisitos de eficiência para todos os municípios (SOUSA; STOŠIĆ, 2005). 
Para identificar possíveis outliers, bem como construir um ranking de eficiência para as DMU, Andersen e Petersen (1993) propuseram o modelo DEA-Supereficiência, que, diferentemente do modelo clássico DEA-VRS, possibilita ao escore de eficiência obter valores superiores à unidade $\left(\theta_{\mathrm{i}}>1\right)$, conforme exposto em (2).

$$
\begin{gathered}
\min \mathrm{E}_{\mathrm{j}}-\delta \mathrm{e}^{\prime} \mathrm{s}^{-}-\delta \mathrm{e}^{\prime} \mathrm{s}^{+} \\
\text {s. a. : } \mathrm{E}_{\mathrm{j}} \mathrm{X}_{\mathrm{j}}=\sum_{\substack{k=1 \\
k \neq j}} \mathrm{z}_{\mathrm{k}} \mathrm{X}_{\mathrm{k}}+\mathrm{s}^{-} ; \mathrm{Y}_{\mathrm{j}}=\sum_{\substack{k=1 \\
k \neq j}} \mathrm{z}_{\mathrm{k}} \mathrm{Y}_{\mathrm{k}}-\mathrm{s}^{+} ; \mathrm{Z}, \mathrm{s}^{+}, \mathrm{s}^{-} \geq 0
\end{gathered}
$$

onde $\mathrm{X}_{\mathrm{j}}$ é um vetor input $\mathrm{m}$-dimensional; $\mathrm{Y}_{\mathrm{j}}$ é um vetor outputs-dimensional para o j-ésimo município; $E_{\mathrm{j}}$ é um escalar que define a ação do vetor input para produzir o vetor output, dentro da tecnologia de referência; $Z$ é um vetor de intensidade, onde $\mathrm{z}_{\mathrm{k}}$ representa a intensidade da k-ésima unidade; $\delta$ é um número infinitesimal nãoarquimediano; e' o vetor de linha $(1, \ldots, 1)$ de dimensão apropriada; $\mathrm{s}^{+}$é um vetor de folgas relativo aos outputs e $\mathrm{s}^{-}$é um vetor de folgas relativo aos inputs. Destaca-se que a importância dos modelos de supereficiência, no tocante ao potencial de identificar e eliminar outliers, foi recentemente endossada por Banker e Chang (2006).

A classificação de outlier seguiu a recomendação de Hartman et al. (2001) e Avkiran (2007), que estabeleceram que uma DMU com escores de supereficiência iguais ou superiores $\left(\theta_{\mathrm{i}}=2\right)$ é um outlier em potencial, porque ocasiona um impacto excessivo na fronteira eficiente.

De modo a testar hipótese de igualdade das médias entre os escores de eficiência dos modelos DEA-VRS e DEA-Supereficiência, realizou-se um teste t-StudentWelch. Após a realizaçáo dos testes, construíram-se modelos de regressão para explicar os escores calculados via modelo DEA-Supereficiência. Por meio do método de seleção automática stepwise, foram selecionadas as variáveis explicativas, dentre as seguintes candidatas: densidade demográfica $\left(\mathrm{hab} / \mathrm{km}^{2}\right)$; latitude (graus); longitude (graus); PIB per capita ( $\mathrm{R} \$ \mathrm{hab}$ ) e tamanho populacional ( $\mathrm{n}^{\mathrm{o}}$ hab.), cujos dados foram provenientes do Instituto Brasileiro de Geografia e Estatística (IBGE). O método de seleção stepwise consiste em, a cada etapa, adicionar sistematicamente a variável mais significativa dentre as que estão de fora de modelo ou remover a variável menos significativa dentre as que já estão no modelo. 
Utilizou-se o software Efficiency Measurement System (EMS) para detectar os municípios classificados como outliers. Os softwares EMS e R foram utilizados no cálculo dos escores de eficiência dos municípios paulistas. A distribuição espacial dos escores de eficiência foi realizada com o software TabWin. Posteriormente, utilizando-se o software Gretl, os valores relativos à eficiência foram regredidos em variáveis não discricionárias que pudessem afetar a eficiência das DMU. Em todos os testes estatísticos, foi adotado um nível de significância de 1\% e, para as estimativas intervalares, foram adotados intervalos de $99 \%$ de confiança.

\section{Resultados}

O modelo DEA-Supereficiência permitiu detectar as DMU com dados não plausíveis num contexto real, ou seja, municípios com problemas de informação, com valores de gasto informados ínfimos, ou produção muito acima do esperado. Destaca-se que essas DMU foram excluídas.

A figura 1 apresenta as distribuiçôes de frequência acumuladas dos escores de eficiência dos municípios, segundo o modelo clássico DEA-VRS ( $\mathrm{n}=539)$ e o modelo DEA-Supereficiência $(\mathrm{n}=509)$, além de apresentar a curva com a diferença entre as distribuiçóes. O exame desse gráfico evidencia o impacto da remoção de outliers. Percebe-se que a diferença entre as distribuiçôes cresce até o escore 0.4 e, a partir deste ponto, as duas distribuiçóes se aproximam.

Figura 1. O impacto da remoção de outliers na eficiência $(\Theta)$ dos municípios paulistas

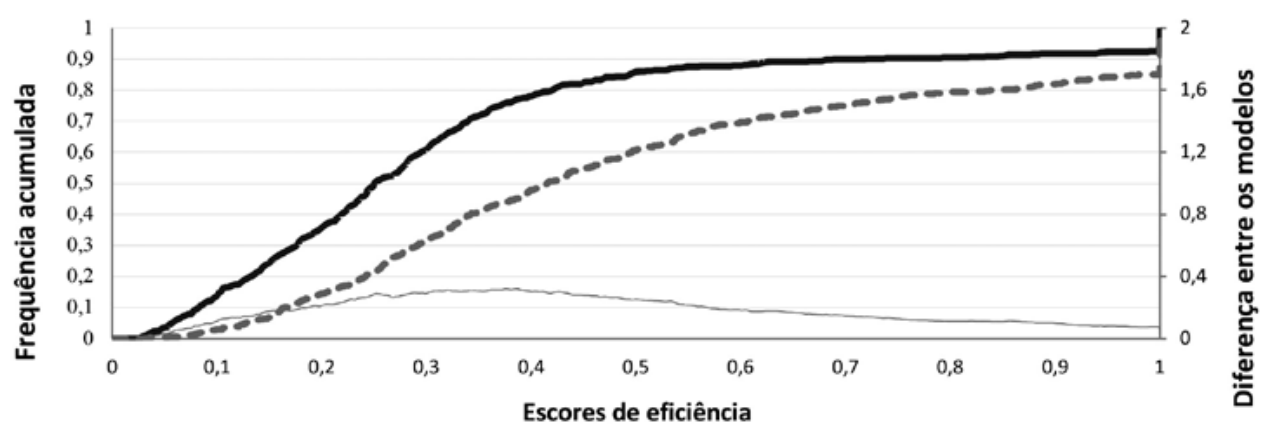

-DEA-VRS - - DEA-Supereficiência —- Diferença entre os modelos

Fonte: resultados da pesquisa (2019). 
A diferença entre as médias dos escores de eficiência com e sem outliers foi igual a 0.170 (IC99\%: 0.127; 0.213). Conclui-se que, uma vez que o valor da diferença média foi negativo, a exclusão dos trinta municípios supereficientes (outliers) tem o efeito de elevar a eficiência média dos municípios restantes. Destaca-se que os outliers concentram-se majoritariamente nas seguintes Regiōes de Saúde (RRAS): (i) RRAS 12 - Araçatuba/São José do Rio Preto, que está situada no noroeste do estado e possui nove outliers e (ii) RRAS 8 - Sorocaba, situada no sul do estado e possui seis outliers.

Tomando-se como referência as estatísticas descritivas (média e desvio-padrão) referentes à eficiência gerada pelo modelo DEA-Supereficiência, foram criadas categorias para os escores de eficiência $(\theta)$ segundo critérios similares aos adotados por Fonseca e Ferreira (2009). Destaca-se que a eficiência média dos municípios paulistas, no tocante aos gastos com Atenção Primária à Saúde, é de 0.488 . Assim, esses resultados indicam que, após a remoção das DMU supereficientes, os municípios investigados poderiam, em média, reduzir os recursos empregados em APS em até $51.15 \%$ (1-0.488), sem reduzir os produtos gerados.

A figura 2 apresenta a distribuição espacial das estimativas de eficiência dos municípios paulistas. Percebe-se a predominância de municípios com ineficiência moderada no tocante à eficiência do gasto em APS. Destaca-se que a variação do escore de eficiência parece estar relacionada à localização geográfica dos municípios.

\section{Figura 2. Distribuição espacial das estimativas de eficiência dos gastos em APS}

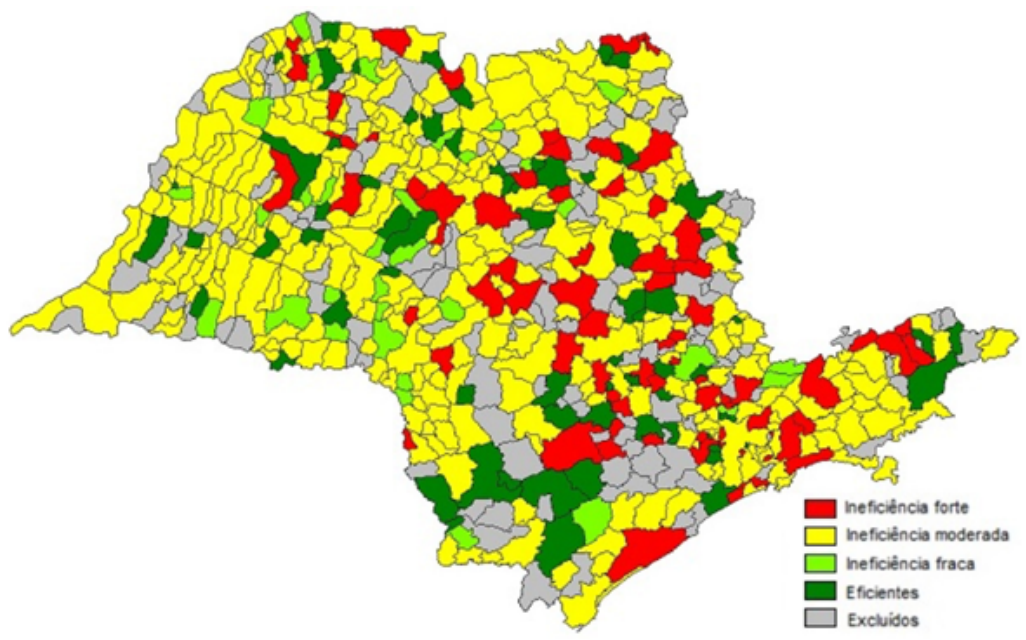

Fonte: resultados da pesquisa (2019). 
A figura 3 apresenta os diagramas de dispersão e a matriz de correlação entre os escores de eficiência do gasto em APS dos municípios paulistas (efic) e as variáveis não discricionárias densidade demográfica (dens); latitude (lat); longitude (long); PIB per capita (pib) e tamanho populacional (pop). Para atenuar o problema de assimetria e peso nas caudas dessas variáveis, conforme sugerido por Greene (2008), tomou-se o logaritmo natural de todas as variáveis, exceto para a latitude e para a longitude. A partir deste procedimento, as variáveis se tornam menos sensíveis a observaçóes discrepantes (outliers). Os histogramas de frequência para cada variável encontram-se na diagonal principal da matriz, que é o locus das entradas onde a correlação é igual a 1 .

Figura 3. Diagramas de dispersão e matriz de correlação entre o escore de eficiência e as variáveis não discricionárias

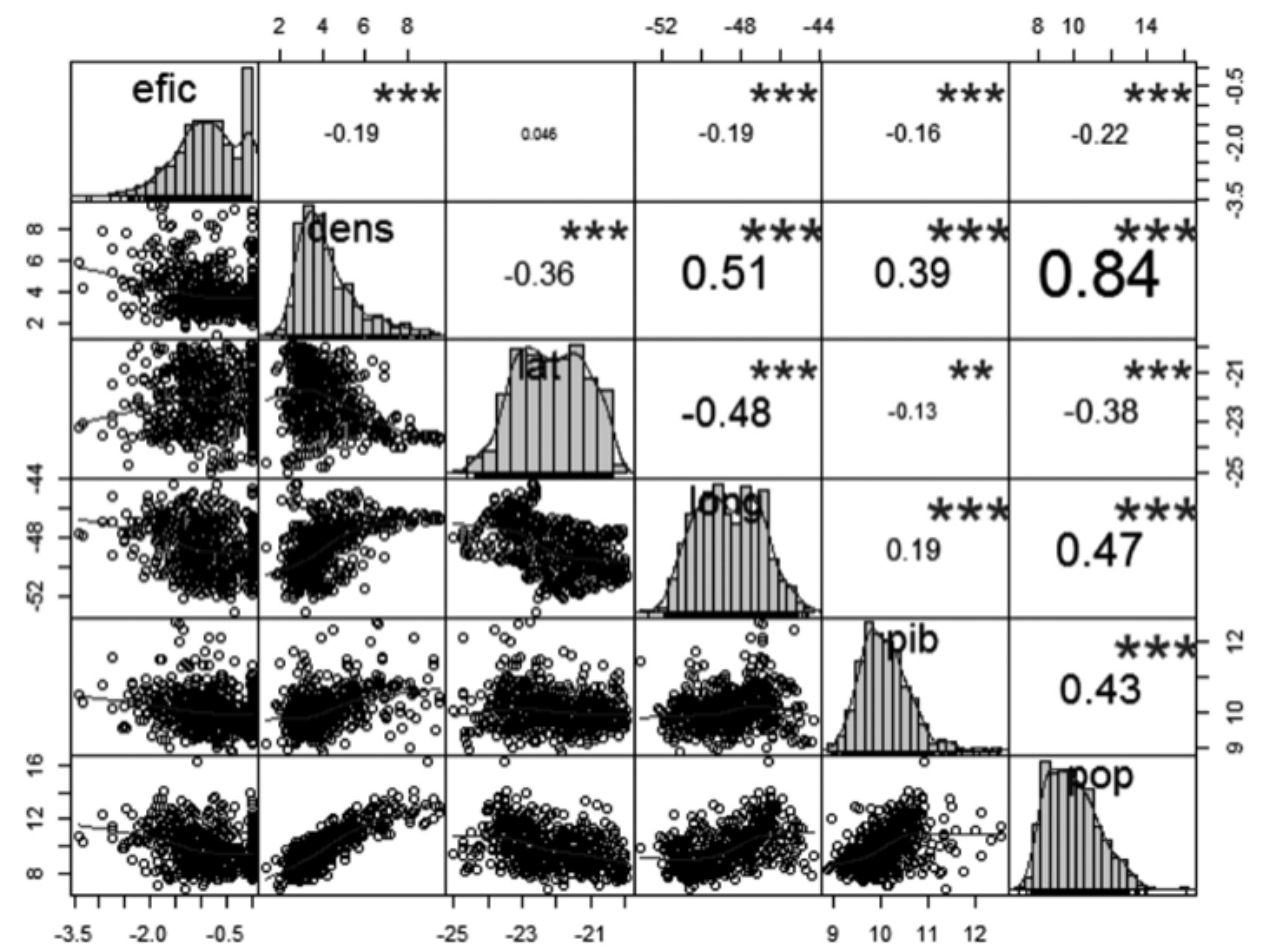

Nota: os asteriscos denotam a significância do teste de correlaçáo de Pearson. A representação do Valor $\mathrm{P}$ é: ${ }^{*} \mathrm{p}<0.10,{ }^{* *} \mathrm{p}<0.05,{ }^{* * *} \mathrm{p}<0.01$.

Fonte: resultados da pesquisa (2019). 
Apesar de as correlações entre a eficiência e as variáveis não discricionárias serem relativamente fracas, percebe-se que todas foram estatisticamente significativas, exceto para a latitude. Em acréscimo, percebe-se que a eficiência é negativamente correlacionada com o tamanho populacional e com a densidade demográfica, e que municípios com maior PIB per capita tendem a ser menos eficientes no gasto em Atenção Primária à Saúde.

Com base nas variáveis explicativas apresentadas na figura 3, construíram-se regressōes por meio do método de seleção automática stepwise. As variáveis "densidade demográfica" e "população" não foram inseridas em uma mesma equação, uma vez que são fortemente correlacionadas. Os resultados dos modelos ajustados são apresentados na tabela 1. Percebe-se que o Modelo 4 possui o melhor ajuste segundo os critérios de seleção de Akaike e Schwarz, entretanto, o coeficiente de determinação calculado é inferior a dez por cento. Diante do exposto, fica evidente que o poder preditivo do modelo é baixo. Não foram encontradas evidências de que a eficiência do gasto público se relacione com o PIB per capita dos municípios paulistas. Por outro lado, a eficiência é inversamente proporcional ao tamanho populacional e à longitude para o nível de significância de $1 \%$.

Tabela 1. Determinantes da eficiência $(\theta)$ dos municípios do estado do Rio de Janeiro via seleção automática stepwise em 2015

\begin{tabular}{lcccc}
\hline & 1 & 2 & 3 & 4 \\
\hline Ln (dens) & $-0.067^{* * *}$ & & $-0.044^{*}$ & \\
& $(-0.021)$ & & $(0.024)$ & \\
& & & & \\
Ln (pib) & -0.118 & $-0,093$ & $-0.118^{* *}$ & -0.090 \\
& $(-0.056)$ & $(0,057)$ & $(0.056)$ & $(0.057)$ \\
& & & & \\
Ln (pop) & $-0.084^{* * *}$ & & $-0.068^{* * *}$ \\
& & $(0,022)$ & & $0.024)$ \\
Latitude & & & $-0,05$ & $-0.053^{*}$ \\
& & & $(0.028)$ & $(0.028)$
\end{tabular}

continua... 


\begin{tabular}{lcccc}
\hline & 1 & 2 & 3 & 4 \\
\hline Longitude & & & $-0.061^{* * *}$ & $-0,058^{* * *}$ \\
& & & $(0.021)$ & $(0.020)$ \\
\hline R-quadrado ajustado & 0,041 & 0,049 & 0,054 & 0,062184 \\
Estatística F & 11,793 & 14.226 & 8,227 & 9,421 \\
P-valor (F) & 0,000 & 0,000 & 0,000 & 0,000 \\
Critério de Akaike & 1009.760 & 1005.104 & 1004.753 & 1000.247 \\
Critério de Schwarz & 1022.457 & 1017.801 & 1025.915 & 1021.409 \\
Log da verossimilhança & -501.880 & -499.552 & -497.377 & -495.123 \\
\hline
\end{tabular}

Nota: o erro está representado entre parênteses. A representação do Valor P é: ${ }^{*} \mathrm{p}<0.10,{ }^{* *} \mathrm{p}<0.05$, *** $\mathrm{p}<0.01$.

Fonte: resultados da pesquisa (2019).

\section{Discussão}

$\mathrm{O}$ uso da DEA é recorrente em pesquisas relacionadas à saúde. Como exemplos, podem-se citar os trabalhos de Gonçalves et al. (2007); Rabetti e Freitas (2011), Scaratti e Calvo (2012) e Lobo et al. (2016). O presente estudo se difere dos supracitados, pois, além de mensurar a eficiência do gasto público em atenção primária à saúde dos municípios, examinou sua distribuição espacial. A exemplificação do presente modelo centrou-se nos municípios paulistas, uma vez que fazem parte do estado mais rico do Brasil, e que possui o segundo maior número de municípios do país. Em acréscimo, o artigo utilizou modelos DEA-Supereficiência, consagrados pela literatura (SOUSA; STOŠIĆ, 2005), para detectar municípios classificados como outliers, que foram excluídos da amostra, de modo a se obter resultados mais acurados. Por fim, mas náo menos importante, a presente pesquisa fez uso de algo complementar aos trabalhos citados anteriormente (GONÇALVES et al., 2007; RABETTI; FREITAS, 2011; SCARATTI; CALVO, 2012; LOBO et al., 2016), a saber, uma análise de regressão de modo a apresentar variáveis que se relacionam com a eficiência dos municípios paulistas.

Os resultados obtidos neste artigo corroboram a necessidade de maior controle e fiscalização nos gastos em saúde, conforme evidenciado pelo World Bank (2017). Em especial, este trabalho indicou, no âmbito do estado de São Paulo, a predominância de municípios com ineficiência moderada. Ainda assim, esses municípios deveriam, 
para serem classificados como eficientes, reduzir em média os recursos empregados em APS em até $51.15 \%$, sem alterar o nível de produtos gerados. O artigo também verificou que os escores de eficiência variaram negativamente em função da longitude; logo, municípios da regiáo Leste do estado de São Paulo tendem a ter maiores níveis de eficiência. Em geral, municípios menos populosos apresentaram maiores níveis de eficiência. Parte destes resultados vão contra os estimados por Dias (2011), pois se verificou que quanto mais populoso é o município, maior é a tendência de que os gastos públicos em saúde sejam ineficientes. Assim, possivelmente, os ganhos de escala observados em outros municípios brasileiros não se aplicam aos municípios do estado de São Paulo.

Especificamente no contexto nacional, a avaliação da eficiência em atenção primária à saúde foi recentemente abordada pelo Banco Mundial (WORD BANK, 2017). Conforme este trabalho, que não apresenta técnicas para a detecção de outliers e restrição aos pesos, o Brasil, em comparação com outros países, possui uma eficiência média dos serviços primários de saúde na ordem de 63\% (dito de outra forma, possui ineficiência de 37\%). Ainda segundo o relatório, para os serviços de atenção secundária e terciária à saúde, a eficiência média é ainda mais baixa: 29\% (WORD BANK, 2017). Isso significa que há possibilidade para melhorar consideravelmente a prestação de serviços de saúde no Brasil, utilizando o mesmo nível de recursos.

É importante ressaltar que o SUS possui notado subfinanciamento; entretanto, há espaço para melhorias na gestão dos gastos e aumento da eficiência no serviço prestado. Destaca-se que os resultados aqui apresentados não podem ser extrapolados para outros estados, uma vez que se limitam a uma amostra específica de municípios do estado de São Paulo. Além disso, os escores de eficiência calculados pela DEASupereficiência apresentam algumas limitaçóes: (i) são influenciados pelo input e outputs selecionados; (ii) o modelo estimado não faz uma distinção entre a importância relativa do input e outputs selecionados.

Assim, como tema para trabalhos futuros, sugere-se a incorporação de julgamentos de especialistas acerca da importância relativa do input e dos outputs do modelo. Para tal, recomenda-se a utilização da abordagem multicriterial acoplada à técnica DEA. ${ }^{1}$

\section{Referências}

ANDERSEN, P.; PETERSEN, N. C. A procedure for ranking efficient units in data envelopment analysis. Management science, v. 39, n. 10, p. 1261-1264, 1993. 
AVKIRAN, N. K. Stability and integrity tests in data envelopment analysis. Socio-Economic Planning Sciences, v. 41, n. 3, p. 224-234, 2007.

BANKER, R. D.; CHANG, H. The super-efficiency procedure for outlier identification, not for ranking efficient units. European Journal of Operational Research, v. 175, n. 2, p. 1311-1320, 2006.

BANKER, R. D. et al. Returns to scale in different DEA models. European Journal of Operational Research, v. 154, n. 2, p. 345-362, 2004.

BOGETOFT, P.; OTTO, L. Benchmarking with Dea, SFA, and R. Springer Science \& Business Media, 2010.

BRAGA, G. B.; FERREIRA, Marco Aurélio Marques; BRAGA, Beatriz Bastos. A Eficiência da Atenção Primária à saúde: avaliando discrepâncias. Administração Pública e Gestão Social, v. 7, n. 2, p. 100-107, 2015.

BRASIL. Constituição da República Federativa do Brasil. Diário Oficial da República Federativa do Brasil, Brasília: Senado Federal, DF, 05 de outubro de 2005. Disponível em: <https://www2.senado.leg.br/bdsf/bitstream/handle/id/508200/CF88_EC85.pdf >. Acesso em: 21 abr. 2019.

. Lei Complementar no 141. Regulamenta o $\$ 30$ do art. 198 da Constituiçâo Federal para dispor sobre os valores mínimos a serem aplicados anualmente pela União, Estados, Distrito Federal e Municípios em açôes e serviços públicos de saúde. Presidência da República, 2012. Diário Oficial da República Federativa do Brasil, Brasília, DF, 13 jan. 2012. Disponível em: <http://www.planalto.gov.br/ccivil_03/leis/LCP/Lcp141.htm>. Acesso em: 21 abr. 2019.

. Ministério da Saúde. Secretaria de Gestão Estratégica e Participativa. Departamento de Articulação Interfederativa. Caderno de Diretrizes, Objetivos, Metas e Indicadores: 20132015. 2014. Secretaria de Gestão Estratégica e Participativa. Disponível em: http://bvsms. saude.gov.br/bvs/publicacoes/caderno_diretrizes_objetivos_2013_2015_2edicao.pdf>. Acesso em: 21 abr. 2019.

. Portaria no 4.279. Estabelece diretrizes para a organização da Rede de Atenção à Saúde no âmbito do Sistema Único de Saúde (SUS). Ministério da Saúde, 2010. Diário Oficial da República Federativa do Brasil, Brasília, DF, 30 dez. 2010. Disponível em: <http://bvsms. saude.gov.br/bvs/saudelegis/gm/2010/prt4279_30_12_2010.html>. Acesso em: 21 abr. 2019.

CABRAL, K. F. D. et al. Análise da Eficiência na Atenção Primária à Saúde sob a Ótica dos Profissionais da Área. Revista de Gestão em Sistemas de Saúde, v. 5, n. 2, p. 71-83, 2016.

CHARNES, A.; COOPER, W. W.; RHODES, E. Measuring the efficiency of decision making units. European journal of operational research, v. 2, n. 6, p. 429-444, 1978.

COOK, W. D.; TONE, K.; ZHU, J. Data envelopment analysis: Prior to choosing a model. Omega, v. 44, p. 1-4, 2014. 
COSTA, A. M.; BAHIA L.; SCHEFFER, M. Onde foi parar o sonho do SUS. Le Monde Diplomatique Brasil, 2013. Disponível em: < https://diplomatique.org.br/onde-foi-parar-osonho-do-sus/ >. Acesso em: 21 abr. 2019.

DE SOUSA, M. C. S.; STOŠIĆ, B. Technical efficiency of the Brazilian municipalities: correcting nonparametric frontier measurements for outliers. Journal of Productivity analysis, v. 24, n. 2, p. 157-181, 2005.

DIAS, R. H. Eficiência da atenção primária a saúde nos municípios brasileiros. 2011. Dissertação (Mestrado em Ciências Econômicas) - Universidade de Brasília, Brasília, 2011.

DUARTE, J. M. S. et al. A eficiência dos gastos públicos nos serviços de saúde municipal. In: XVI Congresso USP de Controladoria e Contabilidade. Anais... São Paulo/SP, 2016.

FERREIRA, C. M. D. C.; GOMES, A. P. Introdução à análise envoltória de dados: teoria, modelos e aplicações. 1rd rev. ed. Viçosa: UFV, 2009. 389 p.

FONSECA, P. C.; FERREIRA, M. A. M. Investigação dos níveis de eficiência na utilização de recursos no setor de saúde: uma análise das microrregióes de Minas Gerais. Saúde e Sociedade, v. 18, p. 199-213, 2009.

GOMES, F. B. C. Impasses no financiamento da saúde no Brasil: da constituinte à regulamentação da emenda 29/00. Saúde em debate, v. 38, p. 6-17, 2014.

GONÇALVES, A. C. et al. Análise Envoltória de Dados na avaliação de hospitais públicos nas capitais brasileiras. Revista de saúde pública, v. 41, p. 427-435, 2007.

GREENE, W. H. The econometric approach to efficiency analysis. The measurement of productive efficiency and productivity growth, v. 1, n. 1, p. 92-250, 2008.

HARTMAN, T. E.; STORBECK, J. E.; BYRNES, P. Allocative efficiency in branch banking. European Journal of Operational Research, v. 134, n. 2, p. 232-242, 2001.

LOBO, M. S. C., et al. Dynamic network data envelopment analysis for university hospitals evaluation. Revista de Saúde Pública, v. 50, p. 22, 2016.

MITRE, S. M.; ANDRADE, E. I. G.; COTTA, R. M. M. Avanços e desafios do acolhimento na operacionalização e qualificação do Sistema Único de Saúde na Atenção Primária: um resgate da produção bibliográfica do Brasil. Ciência \& Saúde Coletiva, v. 17, p. 2071-2085, 2012. MÔNICA, K. et al. A PEC dos gastos e o SUS. Revista Conjuntura Econômica, v. 71, n. 1, p. 32-34, 2017.

OZCAN, Y. A., et al. Health care benchmarking and performance evaluation. Springer US, 2008. PAIM, J. S. Modelos de atenção à saúde no Brasil. In: GIOVANELA, L. et al. (Orgs.). Políticas e sistemas de saúde no Brasil. Rio de Janeiro: Fiocruz, p. 88-115, 2008.

. The citizen constitution and the 25th anniversary of the Brazilian unified National Health System (SUS). Cadernos de saúde pública, v. 29, n. 10, p. 1927-1936, 2013. 
RABETTI, A. C.; FREITAS, S. F. T. Avaliação das ações em hipertensão arterial sistêmica na atenção básica. Revista de Saúde Pública, v. 45, p. 258-268, 2011.

SANTOS, L.; CAMPOS, G. W. S. SUS Brasil: a região de saúde como caminho. Saúde e Sociedade, v. 24, p. 438-446, 2015.

SCARATTI, D.; CALVO, M. C. M. Indicador sintético para avaliar a qualidade da gestão municipal da atenção básica à saúde. Revista de Saúde Pública, v. 46, p. 446-455, 2012.

VIEIRA, F. S. Açôes judiciais e direito à saúde: reflexão sobre a observância aos princípios do SUS. Revista de Saúde Pública, v. 42, p. 365-369, 2008.

ZHU, J. Quantitative models for performance evaluation and benchmarking: data envelopment analysis with spreadsheets. Springer, 2014.

WORLD BANK. Um ajuste justo: análise da eficiência e equidade do gasto público no Brasil. Brasília: Relatório Técnico, 2017.

\section{Nota}

${ }^{1}$ TS Gontijo e IA Reis conceberam e projetaram o estudo. TS Gontijo desenvolveu a gestão, exploração e análise dos dados, a elaboração e interpretação dos resultados e a discussão. Todos os autores revisaram criticamente o manuscrito e colaboraram em todo o processo. Todos os autores leram, contribuíram e aprovaram o manuscrito final. 


\section{Abstract}

\section{The determinants of efficiency in Primary Health Care in São Paulo municipalities: a georeferenced model}

This research aimed to measure the efficiency of expenditures on primary health care in the municipalities of São Paulo and to evaluate their correlation with demographic density and population size of municipalities, as well as with their geographic location and GDP per capita. An ex-post, quantitative, and descriptive valuation of the efficiency of SPS spending was carried out for a sample of the municipalities of Sáo Paulo $(n=509)$, considering the data for the year 2015. The present research made use of a Data Envelopment Analysis model with Superefficiency to detect and remove outliers. Finally, the magnitude of the relationship between calculated efficiency scores and non-discretionary variables was verified. The average of the efficiency scores after the exclusion of the outliers municipalities became greater (IC99\%: 0.127; 0.213). On average, São Paulo municipalities are moderately inefficient in terms of spending on APS $(\Theta=0.488)$. The municipalities located in the East range of the State of São Paulo tend to have higher levels of efficiency and that the efficiency is inversely proportional to the population size. The SUS has noticed underfunding; however, there is room for improvements in the management of expenses and increase efficiency in the service provided.

> Keywords: Primary Health Care; efficiency; robust scores; determinants. 